\title{
BOUNDARY-FORCED SPATIAL CHAOS
}

\author{
by \\ Víctor M. Eguíluz, Emilio Hernández-García, Oreste Piro \\ Instituto Mediterráneo de Estudios Avanzados IMEDEA ${ }^{1}$ \\ CSIC-Universitat de les Illes Balears, E-07071 Palma de Mallorca (Spain)
}

\begin{abstract}
We show that the presence of undulated boundaries can induce the formation of spatially chaotic, stationary, and stable structures in models as simple as the Fisher-Kolmogorov equation, which does not display any kind of chaos under common boundaries.
\end{abstract}

\section{Introduction}

In the past few decades, considerable understanding of the phenomenon of temporal chaos in dynamical systems of few degrees of freedom has been achieved[1]. On the other hand, spatiotemporal chaos in extended dynamical systems with infinitely many degrees of freedom is currently under very active investigation[2]. It is remarkable however, that an area of problems laying somehow between the two extremes has not received so much attention, namely, purely spatial chaos as a stationary attractor of extended dynamical systems $[3,4,5,6,7,8,9]$.

In the context of fluid dynamics, the existence of spatially chaotic, but temporally steady solutions would also fill a conceptual gap between two well-studied complex phenomena: Lagrangian chaos, and Eulerian chaos or turbulence. The former refers to the chaotic motion of a fluid parcel which might occur in flows that are not necessarily chaotic in their Eulerian description. In fact, a laminar flow may induce chaotic motion for the fluid particles [11, 12, 13], and in three dimensions this is even possible if the flow is steady. On the other extreme, the road to turbulence is usually associated to a hierarchy of increasingly spatiotemporally chaotic Eulerian velocity fields $\mathbf{v}(\mathbf{r})$. Frozen spatial chaos would then refer in this context to a third possibility: a stationary velocity field $\mathbf{v}(\mathbf{r})$ spatially chaotic in the Euler description.

Stationary structures in extended nonlinear dynamical systems in one spatial dimension have been considered in some detail in recent investigations, and spatial chaos found. Rigidly travelling waves with spatial chaotic structure can also be considered as a case of spatial chaos, since it is purely spatial in a moving frame of reference [5, 7]. Such one-dimensional systems are specially suitable to analysis because their steady state configurations, which depend only on the unique spatial coordinate, can be described using results of the theory of low-dimensional dynamical systems, by simply interpreting the spatial coordinate as a fictitious time. In the cases previously studied, spatial chaos appears because the nonlinear dynamical system 'evolving' in

\footnotetext{
${ }^{1}$ URL: http://www.imedea.uib.es/Nonlinear
} 
the space coordinate has dimensionality large enough. The high dimensionality of the equations can be due to a) the presence of high-order spatial derivatives in a single evolution equation as in the cases of Kuramoto-Sivashinsky[8] and Swift-Hohenberg equation [4, 9], b) the coupling of several fields each one satisfying lower order differential equation as in the complex GinzburgLandau equation[5] which supports chaotic travelling waves, or c) explicit space dependent forcing terms as in the driven KdV-Burgers [10] equation.

Stationary two-dimensional spatial chaos is basically unexplored at present. In this case application of dynamical system concepts to the analysis of steady state solutions cannot be direct, since we have in principle two equally important spatial coordinates. In addition there is a large variety of possible boundary conditions which surely leads to a variety of steady configurations much richer than in the one-dimensional case. We will be able to show, for a extremely simple model, that rather simple undulated strip-like domain shapes can induce the formation of patterns that are both spatially chaotic and temporally attracting. We believe that the kind of modulated boundaries we propose can be easily implemented in standard experimental pattern formation set-up's such as Faraday waves, convection cells, or open flows. In fact, our work was originally motivated by the observation, in the fluid dynamics experimental setup of a periodic array of pipe bents, that the transversal profile of the steady flow does not necessarily repeat itself with the same periodicity of the array [12].

\section{The model and its boundary conditions}

The connection between the theory of dynamical systems and the study of stationary spatial configurations of one-dimensional extended systems is direct: A stationary pattern satisfies a system of ordinary differential equations with the spatial coordinate as its independent variable which we can think of as a 'time'.

On the contrary, the cases with two or more spatial dimensions can not be tackled along these lines since conventional dynamical systems theory deals with a single time-variable. There are two-dimensional situations, however, where the two independent spatial directions are naturally differentiated by the geometry of the system and we can interpret one of them as playing the role of the time. Thus, the spatial variation in one direction would be interpreted as time evolution of a one-dimensional field that only depends on the remaining spatial coordinate. Symmetries such as parity in a spatial coordinate will appear as time-reversal symmetry after reinterpretation of this coordinate as a time. Particularly suited to our approach will be the case of two-dimensional extended systems evolving in strip-shaped regions much longer (ideally infinite) in the time-like direction than in the space-like one. If the strip is narrow enough, only patterns composed of one or few transverse spatial modes will be allowed and spatial chaos can be readily defined and identified in terms of the usual concepts of dynamical systems theory.

A first remark to be done about this interpretation is that any explicit dependence on the coordinate along the strip amounts to time dependence of the corresponding dynamical system. In particular, if the lateral boundaries of the strip are undulated, the corresponding boundary conditions will translate into time-periodic forcing. 
To focus our attention on spatial chaos induced by boundary effects, we consider a very simple model equation containing only up to second order derivatives and a single field variable known as the Fisher-Kolmogorov (FK) equation:

$$
\partial_{t} \psi=\nabla^{2} \psi+a \psi-\psi^{3}
$$

where $\psi(x, y, t)$ is a real field and $\nabla^{2}$ is the two dimensional Laplacian operator. The real coefficient $a$ in the linear term could be scaled out, but we find convenient to keep it explicit in the equation. The FK equation appears in several contexts ranging from phase transitions (under the name of real Ginzburg-Landau equation, or time-dependent Ginzburg-Landau model [14]) to population dynamics and ecology. Equation (1) has been extensively studied in one and two dimensions.

In one dimension, for systems large enough, most initial field distributions evolve into configurations made of domains where the field takes values near either $\psi_{+}$or $\psi_{-}$, where $\psi_{ \pm}=$ $\pm \sqrt{a}$. These domains are separated by kink or anti-kink-type walls that can move into each other leading to mutual annihilation. By this mechanism, small domains disappear feeding the growth of larger domains whose sizes then increase logarithmically in time until only one of the stationary homogeneous solutions, $\psi_{+}$or $\psi_{-}$, prevailing by chance, takes over the whole system.

In two dimensions, the evolution of a large system leads to coarsening of domains containing either the $\psi_{+}$or the $\psi_{-}$phases, with typical lengths growing as the square root of time[14]. In addition to the weak wall interaction, present as before, the most important driving force for evolution is the tendency to minimize domain wall length. Independently of the dimensionality, the dynamics of (1) is a pure (non inertial) relaxation seeking a minimum of a functional potential[15]. This implies that the asymptotic states can only be fixed points in the functional space, and no limit cycle oscillations nor more complicated attractors can exist.

It was shown by Collet [16] in a more general context that the time evolution and asymptotic states of Eq. (1) in either one or two dimensions are similar to those of an infinite system except within a boundary layer of a size that depends on the $a$ parameter. Therefore, in order to observe the influence of boundaries on pattern evolution we need to consider a domain small enough at least in one of its dimensions. We will consider a strip-shaped domain, elongated in the $x$ direction, which will be called the longitudinal direction, so that the small dimension is the $y$ transversal direction. To be concrete, our domain will be limited by the curves $y_{0}(x)$ and $y_{1}(x)$, where we impose null Dirichlet conditions (that is $\psi\left(x, y_{0}(x), t\right)=\psi\left(x, y_{1}(x), t\right)=0$ ). The choice of Dirichlet boundary conditions is just for convenience but it is not essential for our conclusions. In the one-dimensional case, viewing the stationary solutions of Eq. (1) as orbits of a dynamical system, we immediately rule out chaotic configurations since the dynamical system is just an ordinary differential equation of second order. Chaos can appear, however, if some $x$-dependent periodic forcing is added to the equation. This argument does not apply directly to the two-dimensional case, but it suggests that lateral undulated boundaries (similar to a time-periodic forcing) could induce spatially chaotic structures.

As a particular case we will study the following periodically modulated boundaries:

$$
y_{1}(x)=\frac{d_{1}}{2}(1-\cos (\alpha x)), \quad y_{0}(x)=-1-\frac{d_{0}}{2}(1-\cos (\alpha x+\phi))
$$




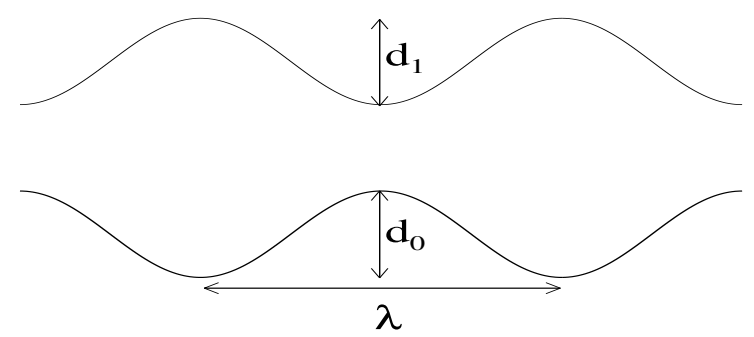

Figure 1: Symmetrically oscillating channel: $\alpha=\frac{2 \pi}{\lambda} ; d_{0}=d_{1}, \phi=0$.

Here $d_{1}, d_{0}$ are the amplitudes of each boundary, $\alpha$ is the spatial frequency, which is the same for both boundaries, and $\phi$ is the phase lag between both boundaries. The case $d_{1}=d_{0}$ and $\phi=0$ gives a channel with symmetrically oscillating width (Fig. 1) whilst if $d_{0}=0$ one boundary is flat. Another interesting case is $d_{1}=d_{0}$ and $\phi=\pi$ that corresponds to having the boundaries in phase, so that the domain has a constant transversal width and undulates along the longitudinal coordinate. Here we will concentrate on the symmetrically oscillating channel, shown in Fig. 1. We stress that we try to remain in the simplest situation that will display spatial chaos. Consideration of more complex models such as the Kuramoto-Sivashinsky or the Swift-Hohenberg equations, which show spatial chaos already with simple boundaries, or more complicated boundaries having for example two incommensurate undulation periods for the upper and lower boundaries (corresponding to a quasi-periodic forcing) could only increase the complexity of stationary solutions.

Boundary conditions in the longitudinal $x$ direction remain to be specified. The analogy with a temporal variable would be the strongest if the region were infinite in that direction, with the only requirement of boundness of the solution. However, an infinite domain is not adequate for the numerical studies that will follow. As a consequence we need to impose periodic boundary conditions (of period $L$ ) along the $x$ direction. In this way we are restricting the class of solutions to periodic orbits of period $L$ or less in the time-like $x$-coordinate. However we can still identify as related to spatial chaos configurations that have the maximal period $L$, provided this period increases as system size $L$ increases, and the periodic orbits approach a chaotic trajectory.

A convenient way to handle the boundaries consists in mapping the region limited by $y_{0}(x)$ and $y_{1}(x)$ (and by $\left.x=0, L\right)$ to a rectangular one: $\tilde{y}_{1}=1, \tilde{y}_{0}=0$ and $x=0, L$. The map $(x, y) \longmapsto(x, \tilde{y})$ for arbitrary functions $y_{0}(x)$ and $y_{1}(x)$ transforms Eq. (1) into an equation for $\tilde{\psi}(x, \tilde{y}, t)=\psi(x, y, t)$ satisfying:

$$
\partial_{t} \tilde{\psi}=\partial_{x x}^{2} \tilde{\psi}+\mathcal{F}(x) \partial_{\tilde{y} \tilde{y}}^{2} \tilde{\psi}+\mathcal{G}(x) \partial_{x \tilde{y}}^{2} \tilde{\psi}+\mathcal{H}(x) \partial_{\tilde{y}} \tilde{\psi}+a \tilde{\psi}-\tilde{\psi}^{3}
$$

where $\mathcal{F}, \mathcal{G}, \mathcal{H}$ depend on the boundary shape $y_{0,1}$.

The new transversal boundary conditions are

$$
\tilde{\psi}(x, \tilde{y}=0)=\psi_{0}(x)=0, \quad \tilde{\psi}(x, \tilde{y}=1)=\psi_{1}(x)=0
$$


Inspection of Eq. (3) tells us that the effect of the boundaries is reflected in the new variables as a parametric forcing.

Making the right hand side of (3) equal to zero to seek for stationary solutions, and thinking of $x$ as time we can look at it as a nonlinear equation for the temporal evolution of a one-dimensional pattern, with a 'time'-periodic parametric forcing due to the boundaries. The present knowledge about spatiotemporal chaos can be used to analyze such dynamics. In particular cases some simple approximations [17] can be carried out to reduce the problem to a simpler one without explicit space dependence.

We could have also performed a conformal transformation, one which conserves the orthogonality of the coordinate lines. The advantage of this kind of transformations is that the Laplacian transforms to itself in the new coordinates but with a space dependent coefficient, i.e. if $T$ is a conformal transformation then $T: \nabla^{2} \longmapsto a(x, y) \nabla^{2}$. Even in this representation the main conclusion would be the same: the non-trivial boundaries affect the dynamical evolution through parametric forcing terms.

\section{$3 \quad$ Numerical Results}

We have performed direct two-dimensional simulations of Eq. (1) in the domain of Fig. 1. Periodic boundary conditions in the longitudinal direction restrict the values of the possible values of the frequency of the boundary oscillation: $\alpha=\frac{2 \pi}{L} n$, being $n$ an integer.

An example of the typical behavior is shown in Fig. 3, where a two-dimensional frozen structure is presented for parameter values $d=1.0, \alpha=\pi, a=20, \phi=0$. The solution consists in the irregular alternation of two phases $\psi_{ \pm}$along the 'time-like' longitudinal direction. Calling 'spatially chaotic' this irregular alternation may raise some doubts. In fact since we are using periodic boundary conditions in the longitudinal direction the configuration shown in Fig. (3) is periodic with the maximum period $L$. However, one can associate to each configuration a sequence of 0's and 1's by taking values of the field at the periodicity $\lambda=\frac{2 \pi}{\alpha}$ imposed by the boundaries (in the same way as one would get a stroboscopic map in the case of a 'external' periodic forcing): if $\psi$ is positive, then one takes the value 1 , if negative, 0 . For example the trajectory in Fig. (2) can be read as '11111110010110100001011'. Our claim is that whatever sequence one could write, it is realizable in this system by only changing the initial condition. Longer sequences would be realized in systems with larger $L$. This establishes the 'spatially chaotic' character of the typical configurations. Other indicators such as the fractal character of Poincaré maps will be discussed in [17]. In the limit of very large systems one could in principle look for Lyapunov exponents and correlation integrals [9].

Our claim can be proved when the domain is a small perturbation of a rectangular one, so that approximations leading to an evolution equation for a single spatial mode can be obtained [17]. In the general case the following physical argument gives an heuristic justification for it: The tendency of the system is to be in a single phase, so that a Ginzburg-Landau energy is minimized [15]. But initial conditions lead to domains of the two phases in competition, with domain walls between them. The tendency to minimize the free energy has two effects: On the 

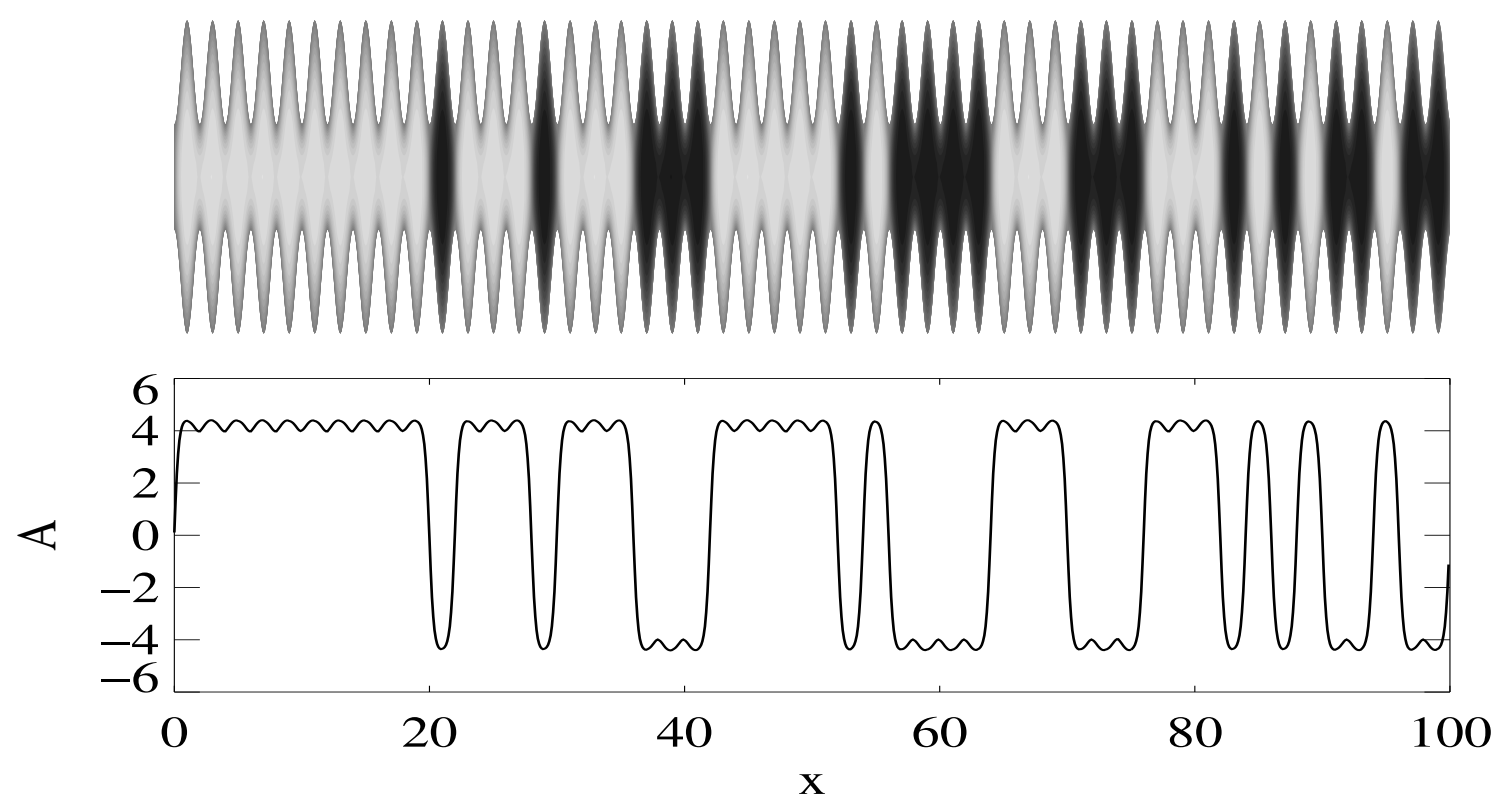

Figure 2: Two-dimensional simulation of the system (1) starting from random initial conditions. The amplitude of the field in the center of the domain is also shown. Parameter values: $d=1.0, \alpha=\pi, a=20, \phi=0$

one hand the walls interact attractively and tend to annihilate by pairs. On the other hand its length tends to be minimal, so that, in our geometry, the walls tend to stay in the places where the distance between the upper and lower boundaries is minimal. If the distance between such places and the amplitude of the boundary oscillations are large enough, this pinning tendency will stop wall annihilation and stabilize configurations such as the one in Fig. 2. This seems to be the origin of the stable disordered structures found. Other geometries, where the distance between both boundaries varies in space, can lead to further interesting behavior.

\section{Conclusions}

We have shown that an undulated boundary can induce stationary, stable, longitudinally chaotic solutions in a model as simple as the Fisher-Kolmogorov equation, which does not display any kind of chaos under common boundary conditions. We can talk about boundary-forced or boundary-induced structures. Spatial chaos should be present in more complex systems, and changing the shape of the boundaries could be a useful way of controlling it.

We are investigating the possibility that these spatially chaotic structures appear in low Reynolds-number flows. Related work on nonlinear extended dynamics influenced by boundaries can be found in [18]. 


\section{Acknowledgements}

We acknowledge financial support from MCyT (Spain) projects CONOCE BFM2000-1108 and BFM2002-04474-C02-01.

\section{References}

[1] H. Bai-Lin, Chaos (World Scientific, Singapore 1990).

[2] M.C. Cross, P.C. Hohenberg, Science 263, 1569 (1994)

[3] P. Coullet, C. Elphick, and D. Repaux, Phys. Rev. Lett. 58, 431 (1987).

[4] E. Hernández-García, M. San Miguel, R. Toral, and J. Viñals, Physica D 61, 159 (1992).

[5] R. Montagne, E. Hernández-García, M. San Miguel, Phys. Rev. Lett. 77, 267 (1996); R. Montagne, E. Hernández-García, A. Amengual, M. San Miguel, Phys. Rev. E 56, 151 (1997).

[6] N.J. Balmforth, Annu. Rev. Fluid Mech. 27, 335 (1996).

[7] H-C. Chang, Annu. Rev. Fluid Mech. 26, 103 (1994).

[8] Y.A. Demekhin, G.Yu. Tokarev, V.Ja. Shkadov, Physica D 52, 338 (1991)

[9] M.I. Rabinovich, A.L. Fabrikant, and L. Sh. Tsimring, Sov. Phys. Usp. 35, 629 (1992).

[10] Malkov, Physica D 95, 62 (1996).

[11] S.W. Jones, O.M. Thomas, H. Aref, J. Fluid Mech. 209, 335 (1989)

[12] Y. LeGuer et al., Experimental study of chaotic advection in a twisted duct flow, preprint.

[13] J.H.E. Cartwright, M. Feingold, and O. Piro, in Proceedings of the NATO ARW "Mixing, Chaos, and Turbulence", H. Chaté and E. Villermaux, editors, (1997).

[14] J. D. Gunton, M. San Miguel and P.S. Sahni, The dynamics of first order phase transitions in Phase Transitions and Critical Phenomena, vol. 8. C. Domb and J. L. Lebowitz, Eds. (Academic Press, New York, 1983).

[15] R. Montagne, E. Hernández-García, M. San Miguel, Physica D 96, 47 (1996); M. San Miguel, R. Montagne, A. Amengual, E. Hernández-García, in Instabilities and Nonequilibrium Structures V, E. Tirapegui and W. Zeller, Eds. (Kluwer Academic Publishers, Dordrecht, 1996).

[16] P. Collet, Nonlinearity 7, 1175 (1994). 
[17] V.M. Eguíluz, E. Hernández-García, O. Piro, S. Balle, Phys. Rev. E 60, 6571 (1999).

[18] V.M. Eguíluz, P. Alstrøm, E. Hernández-García, O. Piro, Phys. Rev. E 592822 (1999); V.M. Eguíluz, E. Hernández-García, O. Piro, Int. J. Bif. Chaos 9, 2209 (1999); ibid., Physica A 283, 48 (2000); ibid., Phys. Rev. E 64, 036205 (2001); I. Sendiña-Nadal, V. Pérez-Muñuzuri, V.M. Eguíluz, E. Hernández-García, O. Piro, Phys. Rev. E 64, 046208 (2001). 